Recepción: 20 / 04 / 2017

Aceptación: 20 / 05 / 2017

Publicación: 15 / 07 / 2017

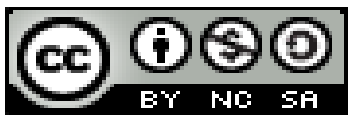

Ciencias de la Educación

Artículo de Investigación

\title{
Impacto del uso de videos educativos complementando las clases presenciales en educación superior
}

\section{Impact of the use of educational videos complementing classroom classes in universities}

\section{Impacto do uso de vídeos educativos complementando as classes reais no ensino superior}

\author{
Francisco R. Riccio-Anastacio ${ }^{\mathrm{I}}$ \\ francisco.riccioa@ug.edu.ec \\ Carlos J. Molestina-Malta ${ }^{\text {II }}$ \\ carlos.molestinam@ug.edu.ec \\ Mayra A. Veliz-Ozaeta III \\ mayra.velizo@ug.edu.ec
}

Correspondencia: francisco.riccioa@ug.edu.ec

\footnotetext{
I. Ingeniero en Electrónica y Telecomunicaciones, Máster en Tecnología de los Sistemas de Energía Solar Fotovoltaica. Docente en la Facultad de Ingeniería Industrial de la Universidad de Guayaquil, Guayaquil, Ecuador.

II. Ingeniero Industrial, Magister en Sistemas de Producción y Productividad. Docente en la Facultad de Ingeniería Industrial de la Universidad de Guayaquil, Guayaquil, Ecuador.

III. Estudiante de la Facultad de Ingeniería Industrial de la Universidad de Guayaquil, Guayaquil, Ecuador.
} 


\section{Resumen}

El desarrollo de las tecnologías de la información y la comunicación (TIC) permite que los docentes tengan a disposición una gran variedad de herramientas informáticas para enriquecer sus cátedras, entre estas tenemos los videos educativos que contribuyen a transmitir conocimientos de modo audiovisual. La presente investigación propone, con una metodología activa y experimental, la realización de varios videos educativos de una asignatura específica que serán utilizados como material adicional a la clase presencial por un grupo de estudiantes de ingeniería en la Universidad de Guayaquil. Los estudiantes accederán a estos videos mediante una plataforma streaming de libre acceso desde cualquier computadora con conexión a internet. Se quiere comprobar si su utilización beneficia la asimilación de conocimientos, lo que los convertiría en una herramienta útil para el aprendizaje de la asignatura en cuestión y serviría de base para su aplicación en otras cátedras y en la educación básica o superior en general.

Palabras clave: Video educativo; TIC; prueba t de dos muestras; muestras independientes. 


\begin{abstract}
The development of information and communication technologies (ICT) allows teachers to have a wide variety of computer tools available to enrich their chairs, among which we have the educational videos that contribute to transmit knowledge in an audiovisual way. The present research proposes, with an active and experimental methodology, the production of several educational videos of a specific subject that will be used as additional material to the classroom class by a group of engineering students at the University of Guayaquil. Students will access these videos through a streaming platform of free access from any computer with internet connection. The aim is to verify if their use benefits the assimilation of knowledge, which would become a useful tool for learning the subject in question and serve as a basis for its application in other chairs and in basic or higher education in general.
\end{abstract}

Keywords: Educational video; TIC; t test of two samples; independent samples. 


\section{Resumo}

O desenvolvimento de tecnologias de informação e comunicação (TIC) permite que os professores tem disponível uma variedade de ferramentas para enriquecer suas cátedras, dentre estes têm vídeos educativos que ajudam a transmitir conhecimentos de modo audiovisual. Esta pesquisa propõe uma metodologia ativa e experimental, realização de vários vídeos educativos de um assunto específico que será usado como um atendimento classe adicional por um grupo de estudantes de engenharia para o material Universidade de Guayaquil. Os alunos irão acessar esses vídeos por streaming livre acesso plataforma a partir de qualquer computador com ligação à internet. Você vai querer verificar se o seu uso beneficia a assimilação do conhecimento, o que os tornaria uma ferramenta útil para aprender o assunto em questão e fornecem a base para aplicação em outros cadeiras e educação básica ou superior em geral.

Palavras-chave: Vídeo educativo; as TIC; teste $\mathrm{t}$ de duas amostras; amostras independentes. 


\section{Introducción.}

En los últimos años se ha hecho evidente la necesidad de reformar el sistema educativo y adecuarlo a las exigencias del mundo contemporáneo. En estos cambios se contempla no solamente el rediseño de los contenidos, sino también de las metodologías educativas en concordancia a los cambios que experimenta la sociedad global, lo que ha traído en consecuencia el desarrollo y uso de las TIC en la educación. El potencial que tienen las TIC en el contexto de la educación superior es vasto ya que existe variedad de modalidades en que se puede presentar.

La Organización de las Naciones Unidas para la Educación, Ciencia y la Cultura (UNESCO) promueve la utilización de las Tecnologías de Información y Comunicación (TIC) como medio para ampliar el acceso a la educación de manera democrática y transparente (UNESCO, 2015), sin embargo, compete a los actores de la comunidad educativa el proponer e implementar los mecanismos que sean más adecuados a la realidad del medio en que se encuentra.

Ejemplo de estos cambios son los originados en la Unión Europea con la creación del Espacio Europeo de Educación Superior (EEES), por lo que las universidades debieron examinar las transformaciones que se están produciendo en su entorno y renovaron en gran parte la forma en que estaba estructurada la enseñanza universitaria (Rodríguez Izquierdo, 2010).

En Ecuador también se inició un cambio del Sistema de Educación Superior cuando La Asamblea Constituyente (julio de 2008) expidió el Mandato Constituyente $\mathrm{N}^{\circ} 14$ mediante el cual encargó al CONEA la elaboración de una evaluación del desempeño institucional de todas las IES del Ecuador con la finalidad de depurar el sistema (Rojas, 2011). 
El docente puede usar varios medios para llegar al alumno fuera del aula de clases, en los últimos años apareció el modelo de cursos masivos online, los Massive Open Online Course ( MOOC), los cuales han tenido gran acogida y por consiguiente amplio desarrollo y uno de sus elementos principales son los videos educativos que tienen por característica una duración de pocos minutos (Letón, Luque, Molanes, \& García, 2013).

La forma en que se accede a la información cambió, el desarrollo de las TIC dio como resultado una gran variedad de herramientas que pueden ser utilizadas, sin embargo, por sus características el video tiene un gran potencial para su uso en educación. La utilización del video posibilita difundir material audiovisual, el cual tiene las siguientes ventajas: poder reproducirlo tantas veces como requiera el estudiante, permite al profesor enseñar el uso de herramientas informáticas especializadas al capturar directamente la interfaz de usuario que se visualiza en la pantalla del ordenador y por último, poder acceder a explicaciones de conceptos previos a una clase (Gálvez \& García, 2013).

La definición de video educativo puede ser muy amplia dependiendo del objetivo que busca conseguir el video. Una definición muy acertada para el contexto formativo es la que proporciona (Bravo, 1996) "el video es uno de los medios didácticos que, adecuadamente empleado, sirve para facilitar a los profesores la transmisión de conocimientos y a los alumnos la asimilación de estos". Este estudio se centra en el uso de los videos educativos como herramienta didáctica complementaria para su uso en la universidad, el objetivo es comprobar el beneficio del uso de los videos educativos para la mejor asimilación de contenidos de las asignaturas por parte de los estudiantes. 


\section{Materiales y métodos.}

Para conseguir evidencia acerca del beneficio del uso de videos educativos en la docencia, se desarrolló un experimento donde se definieron dos variables, una independiente y la otra dependiente (Hernández, Fernández, \& Baptista, 2014), la variable independiente son los videos educativos y la dependiente las notas obtenidas en el parcial -periodo de estudio-, que se consideran como indicadores del nivel de aprendizaje logrado (Pablo-Lerchundi, et al., 2013). Para el experimento se utilizaron dos grupos de estudiantes con semejantes condiciones, es decir: mismo docente, misma asignatura, misma carrera, mismo tipo de aula; fijando un grupo experimental que recibió el tratamiento con los videos y otro de control donde está ausente la variable de control.

Lo primero fue elegir una hipótesis que represente adecuadamente la relación entre las variables estudiadas, para el experimento se tomaron dos muestras, una fueron las notas del parcial pertenecientes al grupo de control y la otra las notas del grupo experimental, ambas muestras son independientes ya que las observaciones de una muestra no tienen ninguna influencia con las observaciones de la otra (Newbold, Carlson, \& Thorne, 2008).

Se realizaron cuatro videos educativos en total, los cuales consisten en tutoriales donde se utiliza Excel como herramienta para el análisis y resolución de temáticas importantes (ver Figura 1), de la asignatura Investigación de Operaciones de la carrera Ingeniería Industrial de la Universidad de Guayaquil. La implementación de los videos se puede lograr con pocos recursos, una computadora con cámara y micrófono, más una aplicación de grabación son los elementos básicos que se utilizaron para la producción del material audiovisual. 
Figura 1. Imagen de uno de los videos educativos elaborados

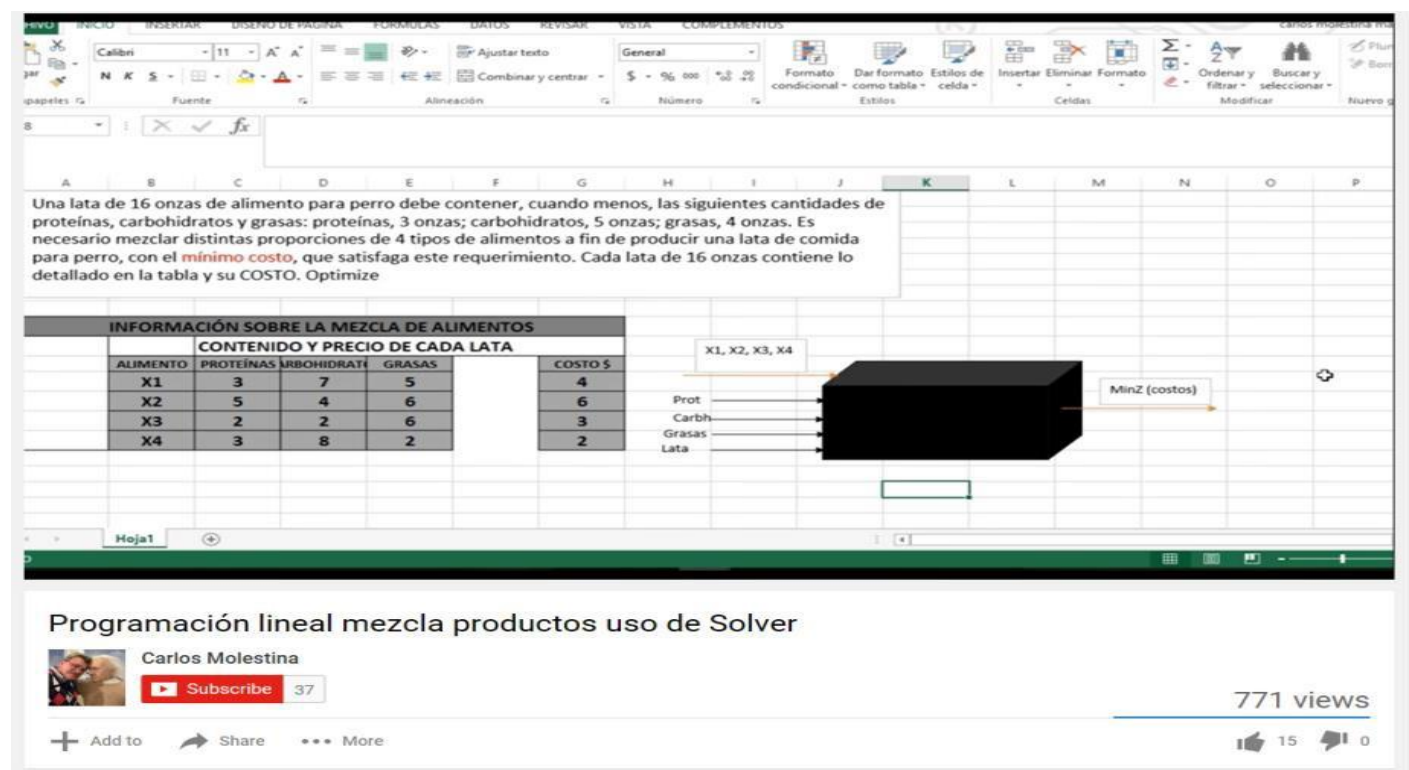

Fuente: Elaboración Propia

Las clases impartidas en el aula por el docente no sufrieron cambios, los videos se utilizaron como material adicional para los estudiantes del grupo experimental. Para este trabajo se consideraron varios aspectos importantes previo a la realización de los videos educativos (García, 2014), como el formato del video, los contenidos y objetivos que se esperan alcanzar. Estos videos representan la variable independiente, y la variable dependiente serán las calificaciones de ambos grupos de estudiantes.

El análisis estadístico para dos muestras independientes que se utilizó fue el contraste de hipótesis de la diferencia entre las medias de las muestras, este procedimiento se basa en la distribución $\mathrm{t}$ para dos muestras independientes. El proceso para comparar dos poblaciones comienza con la formulación de una hipótesis sobre la naturaleza de las dos poblaciones y la 
diferencia entre sus medias, se acepta la hipótesis nula o se rechaza en favor de la hipótesis alternativa (Newbold, Carlson, \& Thorne, 2008).

\section{Resultados.}

Para el análisis estadístico se utilizó el software estadístico Minitab, este nos permite realizar la "Prueba t de dos muestras" con el cual comparamos las medias de dos grupos independientes para determinar la existencia de una diferencia significativa, para esto realiza un análisis con intervalo de confianza y pruebas de hipótesis de la diferencia entre las medias de dos poblaciones (Otamendi \& Chao, 2011).

Definimos la hipótesis nula y alternativa:

Donde corresponde al grupo experimental y atañe al grupo de control.

El promedio de calificaciones de los estudiantes que vieron los videos y el de los que no los vieron es igual.

El promedio de las calificaciones de los estudiantes que vieron los videos es mayor que el de los estudiantes que no los vieron. 
Figura 2. Resultados análisis en Minitab

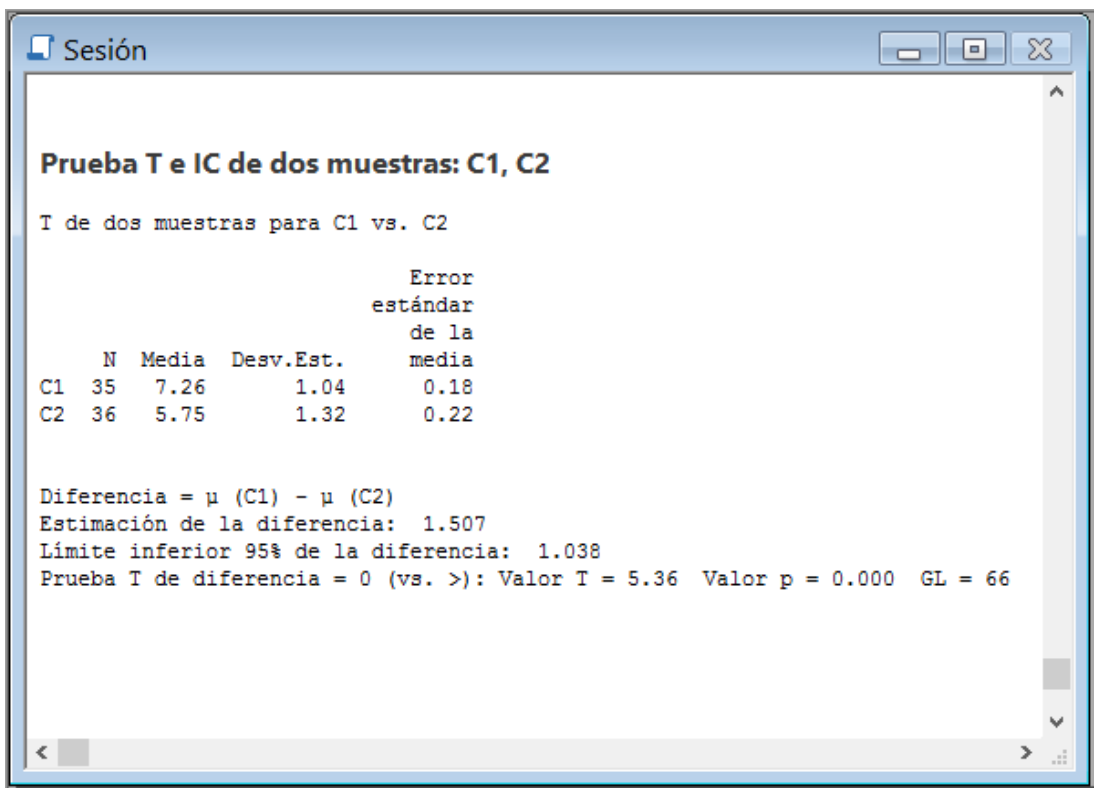

Fuente: Elaboración Propia.

Figura 3. Gráfica de caja de muestras

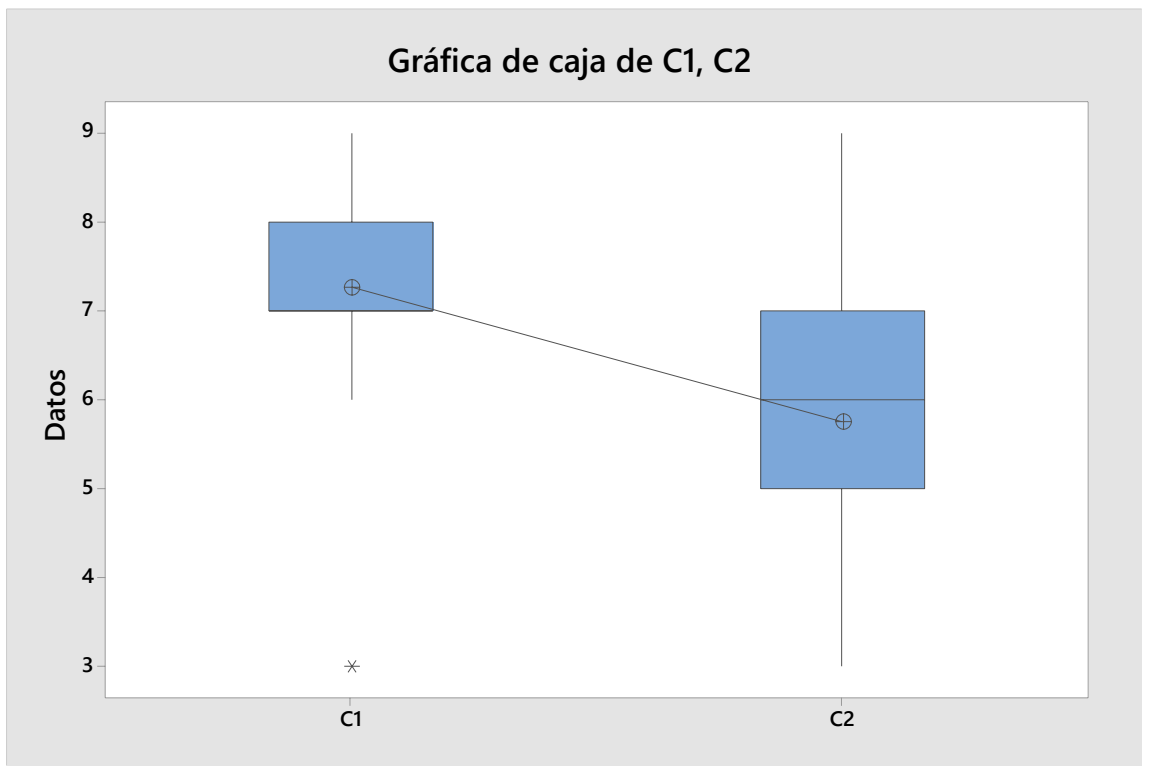

Fuente: Elaboración Propia 
En los resultados obtenidos por Minitab, se observa que el valor $\mathrm{p}=0.000$ por lo tanto se rechaza la hipótesis nula y se acepta la hipótesis alterna. La gráfica de caja nos permite observar las diferencias que tienen las muestras, C1 tiene menor dispersión y su media es mayor.

\section{Conclusiones.}

Se facilita la asimilación de nuevos conocimientos ya que los estudiantes tienen disponibles contenidos relevantes de la asignatura en cualquier momento y no dependerá solamente de la clase impartida en el aula.

El video da a los estudiantes la posibilidad de recapitular la enseñanza del docente las veces que considere necesarias, lo que lo vuelve una fuente de transmisión de conocimientos por parte del docente que podrá ser utilizada en el momento más apropiado a criterio de los estudiantes.

Con el uso del video educativo como material complementario se democratiza el acceso a los contenidos de las asignaturas porque el avance de las TIC ha permitido que puedan ser reproducidos incluso desde un teléfono celular y se compensan problemáticas propias de una clase presencial, que muchas veces se ve afectada por factores como cansancio, falta de concentración, entre otras.

Se comprobó que el uso de videos educativos por parte de los estudiantes del grupo experimental les permitió obtener mejores resultados en las calificaciones de la asignatura que al grupo de control.

Por lo expuesto anteriormente, el uso de los videos educativos constituye una herramienta que complementa adecuadamente las clases impartidas por el docente en el aula. 
El presente artículo se centra en la influencia de los videos en el rendimiento educativo, sin embargo, los promedios atípicos podrían influir en los resultados. Para disminuir la influencia de este factor se debe utilizar un mayor número de grupos como muestra.

\section{Bibliografía}

Bravo, L. (1996). ¿Qué es el video educativo? Comunicar, 100-105.

Gálvez, A. R., \& García, A. R. (2013). Vídeos docentes de Microeconomía: análisis de su impacto externo e interno. Historia y Comunicación Social, 75-84.

García, M. (2014). Uso Instruccional del video didáctico. Revista de Investigación, 43-67.

Hernández, R., Fernández, C., \& Baptista, L. (2014). Metodología de la Investigación. México D.F.: Mc Graw Hill.

Letón, E., Luque, M., Molanes, E., \& García, T. (2013). ¿Cómo diseñar un MOOC basado en mini-vídeos docentes modulares? XVIII Congreso Internacional de Tecnologías para la Educación y el Conocimiento. Madrid.

Newbold, P., Carlson, W., \& Thorne, B. (2008). Estadística para Administración y Economía. Madrid: PEARSON EDUCACIÓN, S.A.

Otamendi, F. J., \& Chao, Á. D. (2011). Estadística para emprendedores: Lecciones prácticas y casos con Minitab. Barcelona: Addlink Software Científico.

Pablo-Lerchundi, I., Núñez del Río, M. C., Saavedra, P., Albéniz, J., Barajas, R., \& Carrillo, I. (2013). Análisis del impacto del uso del vídeo en el laboratorio de química de la titulación de Ingeniería Mecánica en la EUITI. X Jornadas Internacionales De Innovación Universitaria. Madrid: Universidad Europea de Madrid.

Rodríguez Izquierdo, R. M. (2010). El impacto de las TIC en la transformación de la enseñanza universitaria: repensar los modelos de enseñanza y aprendizaje. Teoría de la Educación. Educación y Cultura en la Sociedad de la Información, 11(1), 32-68.

Rojas, J. E. (2011). Reforma universitaria en el Ecuador. Etapa de transición. Innovación Educativa, 11(57), 59-67.

UNESCO. (2015). Directrices para los Recursos Educativos Abiertos (REA) en la Educación Superior. París: Francia.
UNESCO.
(2015).
Las
TIC
la
educación.
Obtenido
de

http://www.unesco.org/new/es/unesco/themes/icts/policy/ 